\title{
Research on the Rice Proteome: The Contribution of Proteomics Technology in the Creation of Abiotic Stress-Tolerant Plants
}

\author{
Setsuko Komatsu
}

Received: 17 May 2008 / Accepted: 26 September 2008 /Published online: 29 October 2008

(C) Springer Science + Business Media, LLC 2008

\begin{abstract}
Proteomics techniques have identified a vast number of proteins that participate in the growth of plants or their adaptation to environmental stresses. Functional analysis of those proteins will contribute to the development of high-yielding crops through artificial manipulation of the basic life phenomena of plants or through the assessment of their stress tolerance. The conditions in almost all farmlands are suboptimal for plant growth, resulting in a calculation that causes the loss to three quarters of the potential yield of crops. Breeding of stresstolerant cultivars has been hampered by the paucity of information on molecular events underlying stress tolerance. A comparative analysis of the response of plants to stress at the protein level, together with physiological measurements, will assist in identifying the genes and pathways that are crucial for stress tolerance. This review examines analyses of the rice proteome under abiotic stress and the potential contribution of proteomics technology in the creation of abiotic stress-tolerant plants.
\end{abstract}

Keywords Rice $\cdot$ Abiotic stress $\cdot$ Proteome $\cdot$ Review

$\begin{array}{ll}\text { Abbreviations } \\ \text { 2D-PAGE } & \begin{array}{l}\text { two-dimensional polyacrylamide gel } \\ \text { electrophoresis }\end{array} \\ \text { MS } & \begin{array}{l}\text { mass spectrometry } \\ \text { polymerase chain reaction }\end{array} \\ \text { PCR } & \begin{array}{l}\text { quantitative trait loci } \\ \text { QTL }\end{array} \\ \text { RuBisCO } & \begin{array}{l}\text { Ribulose-1,5-bisphosphate carboxylase/ } \\ \text { oxygenase }\end{array}\end{array}$

S. Komatsu $(\bowtie)$

National Institute of Crop Science,

Tsukuba 305-8518, Japan

e-mail: skomatsu@affrc.go.jp

$\begin{array}{ll}\text { SOD } & \text { superoxide dismutase } \\ \text { OEE } & \text { oxygen-evolving enhancer protein } \\ \text { PR } & \text { pathogen-related protein } \\ \text { ABA } & \text { abscisic acid } \\ \text { PEG } & \text { polyethylene glycol }\end{array}$

\section{Introduction}

Besides being a very important agricultural crop, rice is useful as a model plant for biological research because it has a smaller genome than those of other cereals [11]. The International Rice Genome Sequencing Project [53] produced a map-based, high-quality sequence that covers $95 \%$ of the $389 \mathrm{Mb}$ genome of rice, including virtually all of the euchromatin and two complete centromeres. The annotation of the rice genome has progressed rapidly [54], and most of the predicted genes are supported by full-length cDNAs [25]. Since the completion of the sequencing of the rice genome, the challenge for the plant-research community has been to identify the function and regulation of rice genes. Unlike the genome, which is static, the proteome is highly dynamic in its response to external and internal cellular events. The responses of the proteome can include changes not only in the relative abundance of various proteins, but also in the post-translational modification of each protein and protein-protein interactions (Reviewed by Komatsu and Yano [35]).

Because the analysis of a protein is the most direct approach to defining the function of its associated gene, proteome analysis linked to genome-sequence information is a very powerful tool in functional genomics [32]. However, the genome and proteome of an organism do not correspond on a one-to-one basis. Alternative transcrip- 
tion initiation and splicing of mRNAs can produce multiple transcripts from a single gene; alternative translationinitiation sites can then produce different proteins from each of these transcripts, and these protein variants can be targeted to different compartments in the cell. Furthermore, post-translational modifications can alter the activity, location, and stability of a protein, and also increase the complexity and dynamics of proteins [36]. Proteins can undergo a staggering array of highly regulated changes that have enormous implications for biological processes, and are the major participants in most processes in living cells. So, proteome research is relevant to the study of organisms at the molecular level.

Many reference maps built by means of two-dimensional polyacrylamide gel electrophoresis (2D-PAGE) catalog the proteins that are expressed in particular organs, tissues, cells, or even sub-cellular compartments in plant (Reviewed by Canovas et al. [6]). Recently, many gel-free proteomics systems have been developed for the purpose of differential proteome analysis. The application of gel-free protein separation methods such as multidimensional protein identification technology (MudPIT), isotope-coded affinity tags (ICAT), and isobaric tags for relative and absolute quantification (iTRAQ) [43] began to be used widely along with the development of the mass spectrometer (MS). Differential proteomics, as technique for identifying functional proteins, requires the ability to detect and quantify changes in proteins. 2D-PAGE coupled MS such as 2Dfluorescence difference gel electrophoresis (DIGE) is highly complementary technique in this respect when applied to proteomic studies [29]. Strategies involving the combined use of 2D-PAGE and MS may lead to the discovery of proteins of agronomic [50] or medical interest [56]. However, a single protein-profiling experiment can provide only a few clues regarding the function of a protein. The integration of the data from such experiments with results from other experiments or from other distinct proteomic approaches, in combination with searches in databases of information on the genome, is critical to extracting useful knowledge from proteome.

To grow and develop optimally, plants need to sense and process information from their surroundings. Because plants are not motile, they have to be highly responsive to environmental changes, including stress conditions. Proteomic approaches that involve identifying the proteins that are differentially regulated in response to environmental conditions are becoming common in post-genomic research in rice. The conditions present in almost all farmlands are suboptimal for plant growth and it has been calculated that about $70 \%$ of the potential yield of crops may be lost as a result of unfavorable environmental conditions [51]. Molecular breeding of stress-tolerant cultivars has been hampered by a shortage of information on the molecular events underlying tolerance to stress. This review describes some initial steps toward determining the physiological significance of some proteins that have been identified by using proteome analysis in rice exposed to a range of abiotic stresses and the contribution of proteomics technology in the creation of stress-tolerant plants will be discussed.

\section{Rice proteome analyses under abiotic stress}

More than $40 \%$ of proteomics studies on stressed crops have been performed on rice [50]; furthermore, more than $50 \%$ of proteomics studied on rice involve salt or cold stresses. Proteomic studies involving several abiotic stresses are listed in Table 1. Plant growth, the level and duration of stress and the phenotype of the plant are the most important factors in the analysis of proteomic changes in stressed plants. The rice proteome database (http:// gene64.dna.affrc.go.jp/RPD/), which is based on 2D-PAGE patterns, provides extensive information on the progress of research on the rice proteome [31]. The database, which is a useful tool, contains information from functional analyses of physiologically significant proteins, such as stressinduced proteins. The information obtainable from the proteome databases is helpful in predicting the function of various proteins; it can also assist in their molecular cloning for functional analysis. The identification of novel genes, the determination of their expression patterns in response to stress, and an understanding of their functions in stress adaptation will provide us with the basis for effective genetic engineering strategies for improving the tolerance of rice to various stresses [28].

The proteome of rice has been studied for 15 years and a previous review highlights the increasing level of success of proteomics in studying this crop at the proteome level [50]. The current review highlights some recent proteomics studies on rice and addresses the range of potential applications of proteomics in the plant science of rice. It also provides an introduction to analyses of the proteome of rice under conditions of abiotic stress, such as drought, salt, cold, or ozone.

Proteomic analyses of rice under drought stress

Drought tolerance is a requirement in plants that experience prolonged deficits in soil water. Tolerant plants can maintain the water content of their tissues, survive a reduction in tissue water content and recover more completely after rehydration. Drought represents one of the most severe limitations on the productivity of rain-fed lowland and upland rice. Hajheidari et al. [18] reported that the efficiency of breeding for increased drought tolerance 


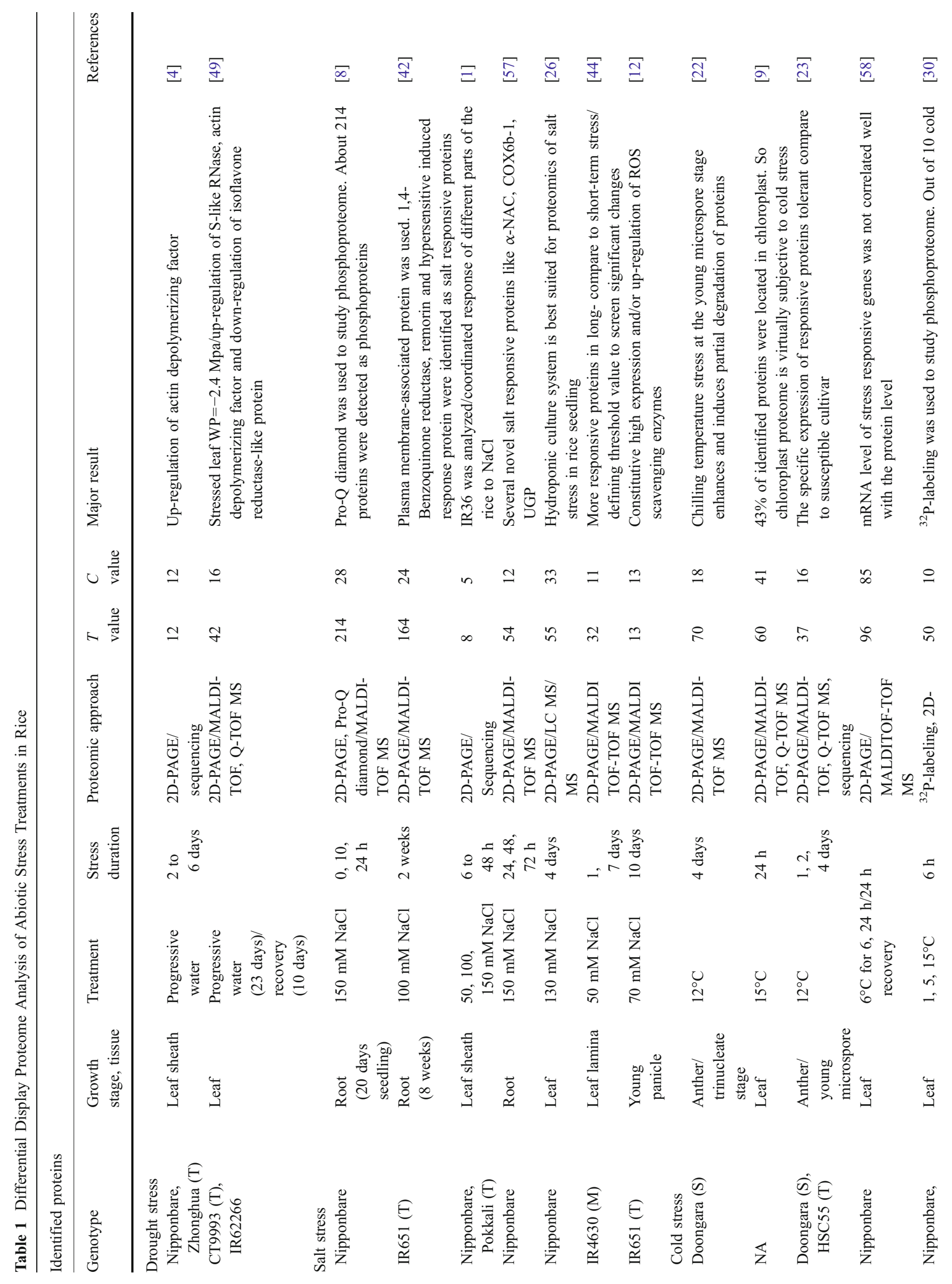




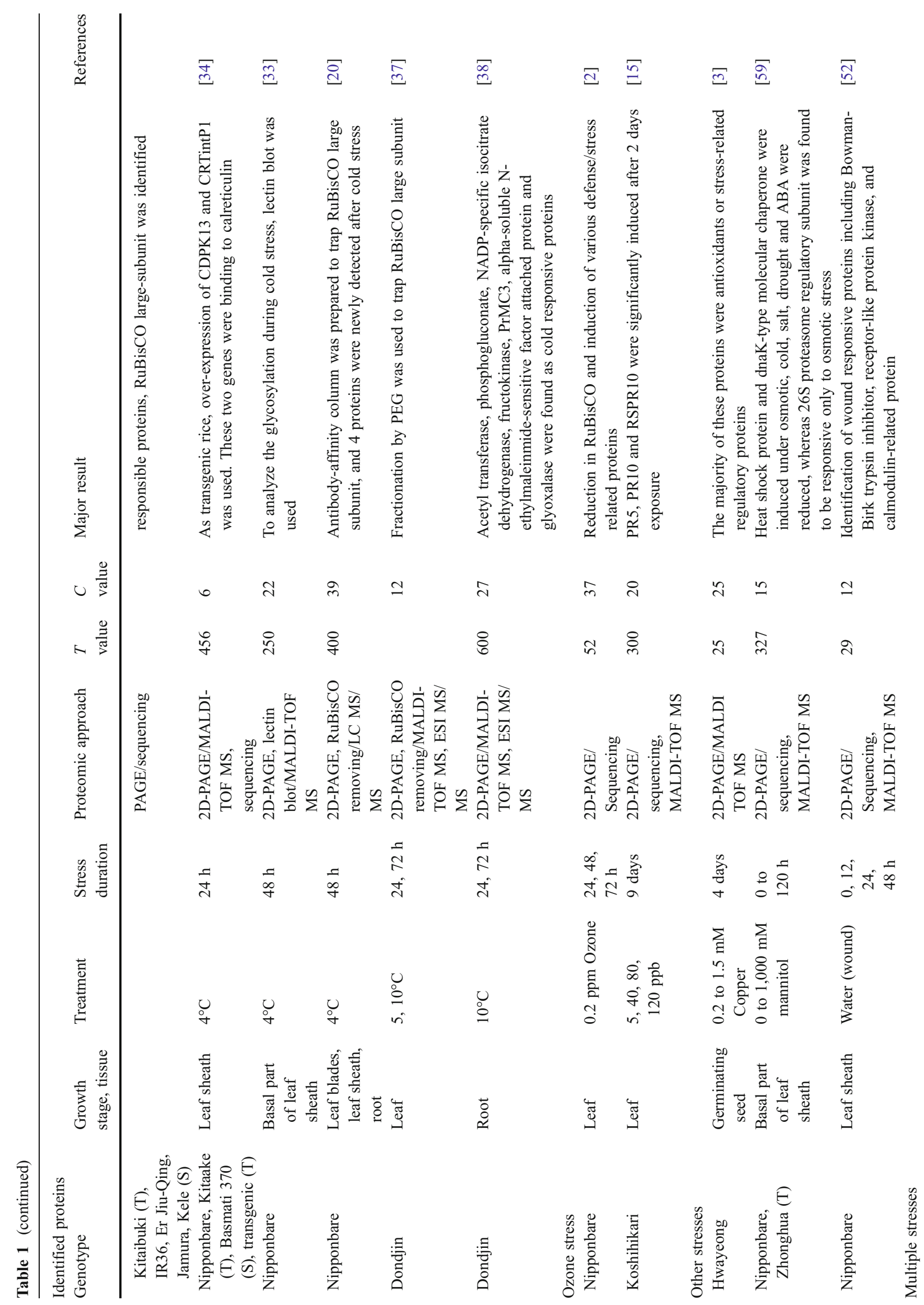




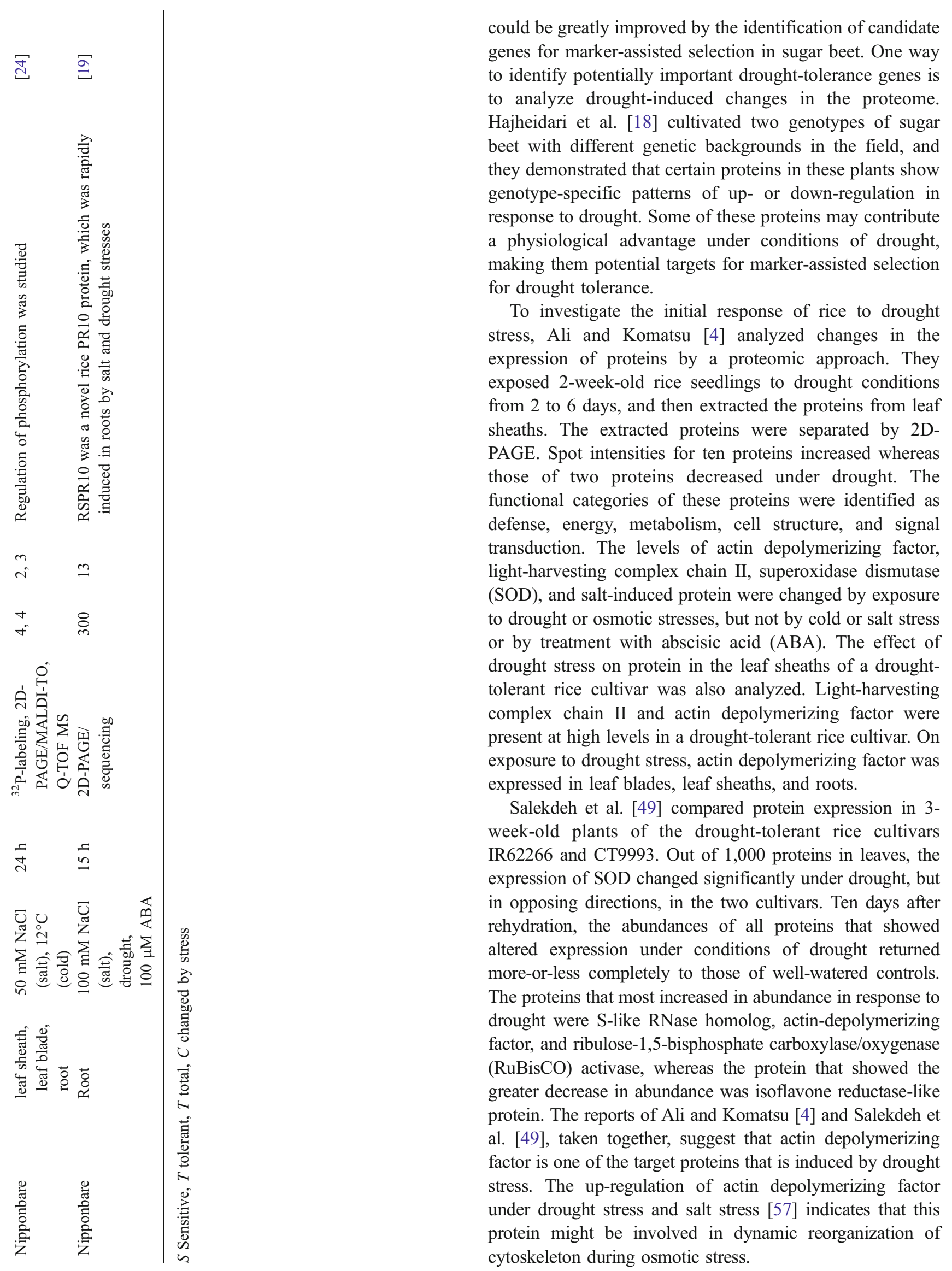


Proteomic analyses of rice under salt stress

Like drought, high salinity also causes a water deficit in plants. Salt stress is one of the major abiotic stresses present in agriculture areas worldwide, with an estimated $20 \%$ of the Earth's land mass and nearly half of all irrigated land being affected by salinity. The increased salinization of arable land is expected to have devastating global effects, with predictions of a $30 \%$ loss of cultivable land within the next 25 years and a loss of up to $50 \%$ by 2050 [57]. Several traditional cultivars, landraces, and wild types of rice, such as Pokkali, CSR types, and Porteresia coarctata appear to be promising materials for the donation of salt-tolerance genes. Many quantitative trait loci (QTLs) for salt tolerance have been identified in rice through the generation of recombinant inbred lines and these are being mapped by using various types of DNA marker [40]. Salt-tolerant transgenic rice plants have been produced by using a wide range of different genes, and transcript profiling by microarray- and macroarray-based methods has opened a route to the discovery of novel salt-stress mechanisms in rice: information from comparative genomics is turning out to be of vital importance in this respect [48].

Abbasi and Komatsu [1] adopted a proteomics approach to identify proteins that increase in abundance in leaf sheathes, roots, and leaf blades of 2-week-old rice seedlings subjected to salt stress. In leaf sheathes exposed to $\mathrm{NaCl}$, eight proteins consistently showed significant changes. Of these eight proteins, three remain unidentified, but the other five were identified as photosystem II oxygen-evolving complex protein, two fructose bisphosphate aldolases, oxygen-evolving enhancer protein (OEE) 2, and SOD. The study also revealed that increased expression of SOD is a common response to cold, drought, salt, and ABA stresses in the leaf sheath. This shows that the accumulation of SOD in response to salt, drought, or cold stresses has a general role in protecting against stress conditions. Under salt stress, enhanced expression of OEE2, aldolase, and photosystem II oxygen-evolving complex protein was detected in the leaf sheathes and leaf blades. SOD and one of the unidentified proteins were also detected in saltstressed leaf sheathes and roots. Another unidentified protein was expressed in leaf sheathes, but was below the limits of detection in leaf blades and roots.

Yan et al. [57] examined the response of 3-week-old rice seedlings exposed to $\mathrm{NaCl}$. More than 1,100 proteins were reproducibly detected, including 34 that were up-regulated and 20 that were down-regulated. Three spots were identified as belonging to the same protein, enolase. Whereas four of the changed proteins had been previously identified as salt stress-responsive proteins, six were novel: UDP-glucose pyrophosphorylase, cytochrome c oxidase subunit 6b-1, glutamine synthetase root isozyme, putative nascent polypeptide-associated complex alpha chain, putative splicing factor-like protein, and putative actin-binding protein. The reports of Abbasi and Komatsu [1] and Yan et al. [57], taken together, show that specific proteins that have enhanced abundances in distinct regions of the rice plant are involved in a coordinated response to salt stress.

Dooki et al. [12] employed a proteomic approach to further elucidate the mechanism of responses of plants to salt at an early reproductive stage. Rice plants were grown in a culture solution and salt stress was imposed at panicle initiation. After 12 days of salt stress, young panicles were collected and the $\mathrm{Na}^{+}$and $\mathrm{K}^{+}$content of panicle tissue and several yield components changed significantly in response to short-term salt stress. The collected panicles were sorted into three different sizes, and their proteome patterns were analyzed by using 2D-PAGE. The expression pattern of 13 proteins significantly changed in all panicle sizes in response to stress and presence of 16 other highly abundant proteins in panicles led to the identification of the proteins that are involved in several salt-responsive mechanisms that may increase the adaptation of plants to salt stress, including higher constitutive expression levels and upregulation of antioxidants and up-regulation of proteins involved in translation, transcription, signal transduction, and ATP generation.

When subjected to salt stress by transferring them to nutrient solution containing $\mathrm{NaCl}$ for 4 days, 18-day-old rice seedlings manifested highly stable and reproducible symptoms, prominent among which were wilting and browning of the third leaf, reduced photosynthetic activity, inhibition in overall seedling growth, and failure to develop a new leaf. Kim et al. [26] used the third leaves as source material for proteomic analysis. A comparison of 2D-PAGE profiles between control and salt-stressed plants revealed 55 differentially expressed spots, of which 47 spots were present in greater abundance than in the control. Most of these identified proteins are involved in major metabolic processes, such photosynthetic assimilation of carbon dioxide and photorespiration, suggesting there is a good correlation between salt stress-responsive proteins and leaf morphology. Moreover, 2D-PAGE immunoblot and enzymatic-activity analyses of third leaves revealed remarkable changes in response to salt stress in the key marker enzymes associated with oxidative damage.

Parker et al. [44] studied rice leaf laminae and found that 32 out of 2,500 proteins were significantly regulated by salinity; so far, 11 of these proteins have been identified by tandem MS. An increase in the abundances of eight proteins, including $\mathrm{RuBisCO}$ activase and ferritin, occurred following $24 \mathrm{~h}$ of exposure to $\mathrm{NaCl}$ and these continued to increase during the following 6 days. Levels of only one protein, a putative phosphoglycerate kinase, were found to increase within $24 \mathrm{~h}$ but not to increase over a longer period 
of exposure to salt. There were also proteins that showed no change $24 \mathrm{~h}$ after exposure to salt, but levels of SOD increased whereas those of $S$-adenosyl-L-methionine synthetase decreased after 7 days of salt treatment.

Nohzadeh et al. [42] extracted the proteome from a root plasma membrane-rich fraction of a salt-tolerant variety of rice, IR651, grown under saline and normal conditions. 2DPAGE analyses showed that 24 proteins were differentially expressed in response to salt stress. Most of the proteins identified are probably plasma membrane-associated proteins, which are known to be involved in several important mechanisms of adaptation of plants to salt stress. These mechanisms included regulation of plasma membrane pumps and channels, membrane structure, oxidative stress defense, signal transduction, protein folding, and the methyl cycle. To investigate the correlation between mRNA and protein levels in response to salinity, quantitative real-time polymerase chain reaction (PCR) analysis of three genes was performed: these genes were salt responsive at the protein level and encoded 1,4-benzoquinone reductase, putative remorin, and hypersensitive induced response protein. No concordance was detected between changes in the levels of gene expression and those of protein expression.

Studies have suggested a critical role of protein phosphorylation in the response of plants to salt stress. However, the phosphoproteome in rice, particularly under salt stress, has not been studied in depth. Chitteti and Peng [8] used Pro-Q Diamond phosphoprotein stain to study differential expression in the phosphoproteome of rice under salt stress. Seventeen differentially up-regulated and 11 down-regulated phosphoproteins were identified. Further analyses indicate that 10 of the 17 up-regulated proteins are probably up-regulated at a post-translational level instead of at the level of the protein concentration: 37 proteins that are differentially regulated in response to salt stress were identified by using SYPRO Ruby stain. Although eight of these are known salt-stress response proteins, the majority have not been reported in the literature.

In proteomic studies, the level of different SOD isoforms were found up-regulated under drought [4, 49], salt [1, 44], and ozone [2, 15]. SOD activity, determined by multigene family, is changing under different environmental conditions. It is a highly effective catalyst mediating a pivotal reaction in the antioxidant pathway [16]. Proteomic studies under stress also provide additional evidence that peroxidase, glutathione-transferase and heat shock proteins are helping in facing stress.

Proteomic analyses of rice under cold stress

Crop plants in tropical and sub-tropical regions are seriously injured by exposure to temperatures below $12^{\circ} \mathrm{C}$ but above the freezing point of water [39]. A primary, if not exclusive, effect of chilling is a phase transition in membrane lipids, which occurs at a critical temperature [39, 47]. Although the way that plants acclimatize to cold stress is not well understood at the biochemical level, it is known that rice seedlings exposed to low temperatures show various changes in their transcriptome. For example, a microarray analysis by Rabbani et al. [46] has showed that 36 genes appear to be induced in rice under cold stress and that the expression level for several genes reached a maximum after $24 \mathrm{~h}$ of cold treatment. Although this geneexpression profiling has deepened our understanding of the response of rice to cold stress, it is still not known how the transcriptional changes are reflected at the translational level. Changes in the transcriptome are not always closely correlated with corresponding changes in protein species [17].

With this limitation in mind, Komatsu et al. [30] performed a proteomic study on rice to gain a better understanding of the molecular mechanisms of acclimatization to cold stress. The phosphorylation of proteins induced by cold stress in rice seedlings was analyzed by 2D-PAGE. Several proteins were found to be phosphorylated upon cold stress, one of which was identified as a fragment of the RuBisCO large subunit. Furthermore, this fragment of the RuBisCO large subunit was found to show a significant increase in phosphorylation in cold-tolerant rice varieties. Compared with rice varieties that grow in tropical or hot regions, those that grow in temperate or cold climates are more responsive to cold stress and RuBisCO large subunit was fragmented and then phosphorylated. Glycosylation is also important in changing the properties and functions of proteins, so a proteomics approach was used, to analyze the importance of glycosylation during cold stress in rice [33]. Proteins extracted from the basal parts of rice leaf sheaths were separated by 2D-PAGE, and subjected to lectin blot analysis using concanavalin A. Cold stress changed the reactivity toward the lectin for 12 of 22 glycoproteins. These proteins were categorized as being involved in energy production. Furthermore, calreticulin, one of the 12 glycoproteins, was also phosphorylated as a result of cold stress.

These results show that cold stress of the basal parts of rice leaf sheaths changes the glycosylation and phosphorylation profiles of calreticulin, a key protein that regulates the quality control of other proteins [33]. The rice calciumdependent protein kinase 13 (CDPK13) and calreticulin interacting protein 1 (CRTintP1) have previously been reported to be related to calreticulin, which is involved in cold stress response in rice. To examine whether or not over-expression of CDPK13 and CRTintP1 would have any effect on the proteins, sense-transgenic rice plants were subjected to proteomic analysis. The 2D-PAGE profiles of 
proteins from a vector control were compared with those of the sense-transgenic rice plants. Two of the proteins that showed differential expression between these lines were calreticulins. Over expression of CDPK13 and CRTintP1 in the cold sensitive cultivar Basmati 370 could protect plants from cold damage [34]. These results suggest that CDPK13, calreticulin, and CRTintP1 may be important signaling components for response to cold stress in rice.

In another study, Cui et al. [9] exposed rice seedlings to a progressively reducing temperature stress treatment involving a shift from the normal growth temperature to cold. Approximately 1,700 protein spots were separated by the 2D-PAGE and 60 protein spots were found to be upregulated in response to the progressively lower temperature treatment. These cold-responsive proteins included two proteins of unknown function, four protein-biosynthesis factors, four molecular chaperones, two proteases, eight enzymes involved in biosynthesis of cell-wall components, seven antioxidative/detoxifying enzymes, and proteins linked to energy metabolism and signal transduction. A large proportion of the proteins $(43.9 \%)$ were predicted to be located in the plastids, implying that the plastid proteome is particularly responsive to cold stress.

Male reproductive development in rice is very sensitive to various forms of environmental stresses, including low temperatures. Cold treatment less than $20^{\circ} \mathrm{C}$ for a few days at the young microspore stage induces severe pollen sterility and thus considerable reductions in grain yield. To investigate this phenomenon, anther proteins were extracted and separated by 2D-PAGE from the coldsensitive cultivar Doongara and the relatively cold-tolerant cultivar HSC55 after cold treatment $[22,23]$. The abundance of 37 proteins changed in anther of Doongara by cold treatment. Among these, one protein was newly induced, 32 protein spots were up-regulated, and four protein spots were down-regulated. Among these 37 protein spots, two antherspecific proteins such as lipid transfer protein and Osg6B were identified; calreticulin was down-regulated and the remaining proteins were up-regulated. Accumulation of these proteins did not vary greatly after cold treatment in panicles of Doongara or in the anthers of HSC55. The newly induced protein named cold-induced anther protein was detected in panicles, leaves, and seedling tissues under normal growth conditions. Quantitative real-time PCR analysis of this mRNA expression showed no significant change between low temperature-treated and untreated plants.

Many of the proteins that are initially identified in proteomic studies are major abundant "housekeeping" proteins involved in fundamental metabolic pathways; proteins expressed at low levels, including regulatory proteins, are harder to detect. This problem is particularly marked in tissues with protein pools that are dominated by a few abundant polypeptide species. For example, RuBisCO has been recognized as the world's most abundant protein [14] and can comprise more than half the total leaf protein in some species. To study proteins at the lower end of the dynamic range, it will be necessary to remove RuBisCO and, possibly, a few other highly abundant chloroplast proteins. Until this becomes possible, a method using high-performance liquid chromatography pre-fractionation to deplete RuBisCO levels in crude leaf extracts provides an interim working solution [55]. This method is useful for detecting proteins that would otherwise not be detectable by conventional multidimensional chromatography/MS or 2DPAGE. Thus, to identify the unique cold-stress response proteins in leaf blades, an antibody-affinity column was prepared that was capable of trapping the RuBisCO large subunit; residual proteins in the flow from the column were subsequently separated by 2D-PAGE [20]. As a result, small changes in stress-responsive proteins could be clearly identified and four proteins were newly detected after cold stress. From the nature of the identified proteins, it was concluded that proteins related to energy metabolism were up-regulated and defense-related proteins were down-regulated by cold stress in leaf blades. These results suggested that energy production is activated in the chilling environment; furthermore, stress-related proteins are rapidly upregulated, whereas defense-related proteins disappear under long-term cold stress.

Lee et al. [37] exposed rice seedlings to temperatures of 5 or $10^{\circ} \mathrm{C}$, and collected them after various time courses. To eliminate the high-abundance proteins such as RuBisCO in leaf tissues, the proteins were fractionated by using polyethylene glycol (PEG). A total 12 up-regulated protein spots were identified, including some novel proteins, which had not previously been identified in reports on proteomic analysis of chilled plants. The identification of some novel low-abundance proteins in response to cold stress may suggest a new homeostasis model that could be used to develop transgenic plants with enhanced cold tolerance [37]. Because the roots are also highly sensitive organs in plants, Lee et al. [38] exposed rice seedlings to cold. To identify the low-abundance proteins in root tissues, samples were fractionated on $15 \%$ PEG and separated by $2 \mathrm{D}-\mathrm{PAGE}$. Multiple dynamic equilibrium adjustments and regulation mechanisms enabled homeostasis to occur. A total of 27 upregulated proteins were identified. Together with the previously identified cold-stress-responsive proteins, a group of novel proteins were identified, including acetyl transferase, phosphogluconate dehydrogenase, NADP-specific isocitrate dehydrogenase, fructokinase, PrMC3, putative alpha-soluble $N$-ethylmaleimide-sensitive factor attachment protein, and glyoxalase 1 . These proteins are involved in several cellular processes, including energy production and metabolism, vesicular trafficking, and 
detoxification. Gene expression at the mRNA level of some selected proteins revealed that transcription levels are not always concomitant with the translational level.

Yan et al. [58] treated 3-week-old rice seedlings at cold and then allowed them to recover. Chilling treatment resulted in stress phenotypes of rolling leaves, increased relative electrolyte leakage, and decreased net photosynthetic rate. The temporal changes in total proteins in rice leaves were examined by using 2D-PAGE. Among approximately 1,000 protein spots reproducibly detected on each gel, 31 protein spots were down-regulated, and 65 were up-regulated at one time point or more. Eighty-five differentially expressed proteins were well-known or novel cold-responsive proteins. Several proteins showed enhanced degradation during chilling stress, especially the photosynthetic proteins, such as the RuBisCO large subunit, of which 19 fragments were detected. The identified proteins are involved in several processes, such as signal transduction, RNA processing, translation, protein processing, redox homeostasis, photosynthesis, photorespiration, and metabolisms of carbon, nitrogen, sulfur, and energy. Gene-expression analysis of 44 different proteins by quantitative real-time PCR showed that the mRNA level does not correlate well with the protein level.

\section{Proteomic analyses of rice under ozone stress}

Ozone is a destructive gaseous pollutant that seriously affects human and animal respiration, as well as causing extensive damage to both natural and cultivated plant populations [7]. The resistance of rice to ozone is a quantitative trait controlled by nuclear genes [27]. The identification of QTLs and the analysis of molecular markers of ozone resistance are important steps in increasing the resistance of rice to ozone stress. Kim et al. [27] reported that QTLs associated with the ozone resistance of rice were mapped on chromosomes by using recombinant inbred lines from a cross between "Milyang 23" and "Gihobyeo", and QTLs were tightly linked to three markers. The association between these markers and ozone resistance in rice cultivars and doubled haploid populations was analyzed. The markers permit the screening of the rice germplasm for ozone resistance and the introduction of resistance into elite lines in breeding programs. The identification of ozone-related QTLs provides an improved understanding of ozone responsiveness in rice, and may lead to applications in breeding for enhanced ozone tolerance.

Agrawal et al. [2] analyzed the responses of plants to ozone by using a proteomics approach. In rice leaves, ozone caused marked visible necrotic damage and enhanced the levels of ascorbate peroxidase proteins; these changes were accompanied by rapid changes in 2D-PAGE. Of a total of 56 proteins investigated, 52 spots were visually identified to be differentially expressed relative to the control. Ozone caused marked reductions in the major photosynthetic proteins in leaves, including $\mathrm{RuBisCO}$, and the induction of various defense- and stress-related proteins. This research provides evidence for the specific and rapid accumulation of certain proteins, such as pathogen-related proteins (PR) 5 and 10, ascorbate peroxidase, SOD, and ATP-dependent caseinolytic protease.

Feng et al. [15] investigated the protein responses in rice seedlings under ambient conditions and under elevated ozone stress by a proteome approach. Twenty proteins were differentially expressed. Expression of the RuBisCO large subunit and $\mathrm{RuBisCO}$ activase were reduced by ozone. The expression of RuBisCO small subunit, RuBisCO large subunit fragment, and OEE 1 and 2 increased at ambient ozone levels and decreased at elevated ozone levels. Ambient ozone levels induced a slight reduction and elevated ozone levels induced a significant reduction in the expression of energy-metabolism proteins. Expression of the antioxidant proteins glutathione transferase and SOD increased upon exposure to ozone, but thioredoxin peroxidase expression decreased. PR5 and two PR10 proteins (PR10/PBZ1 and RSPR10), which are all defense/stressrelated proteins, were significantly induced after 2 days of exposure to elevated ozone, and their levels gradually increased during the exposure period. The results not only indicate a difference in protein responses between leaves exposed to ambient and elevated ozone, but also demonstrate the highly damaging effect of elevated ozone levels on rice seedlings at the proteome level.

\section{Proteomic analyses of rice under other stresses}

Among abiotic stresses, osmotic stress is one of the most severe, and in nature it can be caused by drought, high salinity, or cold or by combinations thereof. Plants respond to osmotic stress at the morphological, anatomical, cellular, and molecular levels. These responses include production of osmolites for osmotic adjustment, synthesis of $\mathrm{Na}^{+} / \mathrm{H}^{+}$ antiporters for ion sequestration, and many other adaptive changes [5]. Although there have been many molecular biological studies of responses to osmotic stress, no reports of studies based on a proteomic approach had been published until Zang and Komatsu [59]. Two-week-old rice seedlings were treated with mannitol. After separation of proteins extracted from the basal part of leaf sheaths by 2DPAGE, 327 proteins were identified. Expression of 12 proteins increased and that of three proteins decreased with increasing concentrations or durations of mannitol treatment. Levels of heat shock protein and dnaK-type molecular chaperone were reduced under osmotic, cold, salt, or drought stresses or on treatment with ABA. In contrast, a $26 \mathrm{~S}$ proteasome regulatory subunit and an 
endosperm luminal binding protein were found to be responsive only to osmotic stress. Levels of glyoxalase I increased in all the tissues examined after osmotic stress. Every protein that accumulated in response to osmotic stress was present at elevated levels in an osmotic stresstolerant cultivar, even in the absence of stress. These results indicated that specific proteins expressed in the basal part of rice leaf sheaths showed a coordinated response to osmotic stress [59].

To eliminate the complex conditions found in the intact plant, Shen et al. [52] used the detached rice leaf sheath, which is a very active part of the rice seedling, for a simple analysis of proteins in wound-response stress. Proteins were extracted from rice leaf sheath 12 to $48 \mathrm{~h}$ after cutting and then separated by 2D-PAGE. Ten proteins were upregulated and 19 proteins were down-regulated. Among these groups of proteins, Bowman-Birk trypsin inhibitor, receptor-like protein kinase, and calmodulin-related protein have been confirmed to be wound-response proteins. The involvement in wound response of the remaining proteins, such as the signal transduction-related proteins, photosynthesis-related protein, and stress-response proteins, cannot be eliminated.

Copper is an essential micronutrient for plants; however, when it is present in high concentrations in soil, copper is also regarded as a major toxicant to plant cells, because of its potential inhibitory effects on many physiological and biochemical processes. Interference with germination-related proteins by heavy metals has not been well documented at the proteomic level. Ahsan et al. [3] examined physiological, biochemical, and proteomic changes in germinating rice seeds in response to copper stress. Germination rates and shoot elongation, plant biomass, and water content following by germination were decreased, whereas accumulation of copper and the thiobarbituric acid-reactive substance content in seeds increased significantly with increasing copper concentrations from 0.2 to $1.5 \mathrm{mM}$. Protein profile analyses by 2D-PAGE revealed that 25 protein spots were differentially expressed in copper-treated samples. Among these, 18 protein spots were up-regulated and seven were down-regulated. The upregulation of some antioxidant and stress-related proteins, and some regulatory proteins, clearly indicated that excess copper generates oxidative stress that may be disruptive to other important metabolic processes. Moreover, downregulation of key metabolic enzymes revealed that the inhibition of seed germinations after exposure to excess copper not only causes starvation in water uptake by seeds, but also results in failure in the reserve mobilization processes. These results indicate that there is a good correlation between the physiological and the biochemical changes in germinating rice seeds exposed to excess copper.
Proteomic analyses of rice under multiple stresses

Khan et al. [24] detected phosphoproteins in rice by in vitro protein phosphorylation followed by 2D-PAGE. Forty-four phosphoproteins were detected on 2D-PAGE after in vitro protein phosphorylation of the crude extract from rice leaf sheath. Among the phosphoproteins detected, 42 were identified through analysis by MS. Most of the identified phosphoproteins are involved in signaling (30\%), and metabolism (18\%). When rice seedlings were treated with various hormones and stresses, it was observed that the phosphorylation of 13 proteins was enhanced differentially by various hormonal or stress treatments. Furthermore, when the hormone/stress-regulated phosphoproteins were compared in the leaf sheath, leaf blade, and root of rice, only cytoplasmic malate dehydrogenase was found to be phosphorylated in all these tissues. In the phosphorylation cascade of rice, glycolytic metabolism processes and $\mathrm{Ca}^{2+}$ signaling appear to be important targets in the response to hormones and stresses. Furthermore, the direct visualization of phosphoproteins by ${ }^{32} \mathrm{P}$-labeling and their identification by MS provides an accurate and reliable method for analyzing the rice phosphoproteome.

Hashimoto et al. [19] investigated stress-response proteins from rice roots using 2D-PAGE and found a rice protein named as RSPR10 induced specifically in roots when 2-week-old rice seedlings were subjected to salt and drought stresses. The full-length cDNA for this protein was cloned and the deduced amino-acid sequence showed a high similarity to the known sequences of PR10, PR10a/ PBZ1, and PR10b. mRNA accumulated rapidly upon exposure to drought, $\mathrm{NaCl}$, jasmonic acid, or probenazole, but not on exposure to low temperatures, ABA, or salicylic acid. This gene was also up-regulated by infection with rice blast fungus. Interestingly, induction was observed almost exclusively in roots. RSPR 10 is a rice PR10 protein that is rapidly induced in roots by salt, drought stresses, or blast fungus infection, possibly through activation of the jasmonic acid signaling pathway, but not by the ABA or salicylic acid signaling pathways.

\section{Conclusion}

In the initial stages of stress response, the change in environmental condition is sensed by the plant and activates a network of signaling pathways. In later stages, the signal transduction pathways activated in the first phase trigger the production of different proteins and compounds that restore or achieve a new state of homeostasis. Qureshi et al. [45] reviewed that owing to gene expression altered under stress, qualitative and quantitative changes in proteins are obvious. The advancement in tools of proteomics has changed the 
whole scenario. Given the high throughput and high sensitivity of MS, coupled via advanced software to protein databases, proteomics is gaining overwhelming response [45]. Proteomics is one of the more powerful techniques among molecular tools that can be used to analyze stressaffected plants. It is well suited to hypothesis-limited research, because it offers an entry point for identifying potentially significant differences in protein levels among plants that received stress. Comparative proteomics using 2D-PAGE involves the identification of proteins that are differentially expressed under different environmental conditions or between wild-type plants and mutants or cultivars. This type of study is useful, because plant lines produced by intensive breeding selection are available that have different degrees of tolerance, which helps in the identification of correlations between responsive genes and observed stress-tolerance phenotypes. This correlation can be verified by analyzing advanced mapping populations, such as recombinant inbred lines, near isogenic lines, or double haploid lines. The application of proteomics in rice breeding is usually initiated by detection of stress-responsive proteins through comparison among stressed plants. Identification of these candidate proteins may then reveal that some of them have functions that are clearly consistent with the stress-tolerance trait. It may be helpful to have a record of proteins as biomarkers.

By using a range of molecular tools, plant scientists are increasingly able to identify and characterize the genes that control key processes and traits in plants. In microarray studies, many transcription factors were found up-regulated under drought, salt, and cold [10, 13, 21, 41, 46]. Microarray analysis is potential tool; however mRNA and protein levels cannot be correlated due to the inability of total mRNA to translate into protein. Although microarrays for transcriptome can be used to detect gene expression in organs, it is the array of proteins that are found within the cell, together with their modifications and interactions, which hold the key to understanding of biological systems. The proteomic technique is therefore an important tool for gene discovery and molecular breeding. Proteomics study using 2D-PAGE has provided new dimensions to assess the changes in protein types and their expression levels under stress. The technique can be further optimized for use in rice proteomics research, for example, by differential analysis using gel-free methods, by using protein arrays for protein-protein interaction, or by organelle purification $[29,35]$. Whereas proteomics can be used in cloning of unique genes by means of differential analysis, the technique is also useful in studying post-translational modifications that can affect the activity and binding of a protein and alter its role within the cell. By the use of completely analyzed genes and the analysis of the functions of their encoded proteins, proteomics is expected to have a marked impact on breeding of new strains of rice. In the near future, a tight correlation should be attainable between proteomics data and other data from comprehensive analyses. These data will provide further in-depth characterization of the proteome of rice and its subcellular compartments, and has the potential to identify new targets for improvements in rice crops.

Acknowledgements This original work was supported by a grant from the rice genome project, Ministry of Agriculture, Forestry, and Fisheries, Japan.

\section{References}

1. Abbasi F, Komatsu S. A proteomic approach to analyze saltresponsive proteins in rice leaf sheath. Proteomics 2004;4:207281 .

2. Agrawal GK, Rakwal R, Yonekura M, Kubo A, Saji H. Proteome analysis of differentially displayed proteins as a tool for investigating ozone stress in rice (Oryza sativa L.) seedlings. Proteomics 2002;2:947-59.

3. Ahsan N, Lee DG, Lee SH, Kang KY, Lee JJ, Kim PJ, et al. Excess copper induced physiological and proteomic changes in germinating rice seeds. Chemosphere 2007;67:1182-93.

4. Ali GM, Komatsu S. Proteomic analysis of rice leaf sheath during drought stress. J Proteome Res 2006;5:396-403.

5. Bohnert HJ, Nelson DE, Jensen RG. Adaptations to environmental stresses. Plant Cell 1995;7:1099-111.

6. Canovas FM, Dumas-Gaudot E, Recorbet G, Jorrin J, Mock HP, Rossignol M. Plant proteome analysis. Proteomics 2004;4:28598.

7. Chameides WL, Saylor RD, Cowling EB. Ozone pollution in the rural United States and the new NAAQS. Science 1997;276:916.

8. Chitteti BR, Peng Z. Proteome and phosphoproteome differential expression under salinity stress in rice (Oryza sativa) roots. J Proteome Res 2007;6:1718-27.

9. Cui S, Huang F, Wang J, Ma X, Cheng Y, Liu J. A proteomic analysis of cold stress responses in rice seedlings. Proteomics 2005;5:3162-72.

10. Dai X, Xu Y, Ma Q, Xu W, Wang T, Xue Y, et al. Overexpression of an R1R2R3 MYB gene, OsMYB3R-2, increases tolerance to freezing, drought, and salt stress in transgenic Arabidopsis. Plant Physiol 2007;143:1739-51.

11. Devos MK, Gale DM. Genome relationships: the grass model in current research. Plant Cell 2000;12:637-46.

12. Dooki AD, Mayer-Posner FJ, Askari H, Zaiee AA, Salekdeh GH. Proteomic responses of rice young panicles to salinity. Proteomics 2006;6:6498-507.

13. Dubouzet JG, Sakuma Y, Ito Y, Kasuga M, Dubouzet EG, Miura $\mathrm{S}$, et al. OsDREB genes in rice, Oryza sativa L., encode transcription activators that function I drought-, high-salt- and cold-responsive gene expression. Plant J 2003;33:751-63.

14. Ellis RJ. The most abundant protein in the world. Trends Biochem Sci 1979;4:241-4.

15. Feng YW, Komatsu S, Furukawa T, Koshiba T, Kohno Y. Proteome analysis of proteins responsive to ambient and elevated ozone in rice seedlings. Agri Eco Environ 2008;125:255-65.

16. Foyer $\mathrm{CH}$, Lenandair M, Kunert KKJ. Photooxidative stress in plants. Physiol Plant 1994;92:696-717.

17. Gygi SP, Rochon Y, Franza BR, Aebersold M. Correlation between protein and mRNA abundance in yeast. Mol Cell Biol 1999;19:1720-30. 
18. Hajheidari M, Abdollahian-Noghabi M, Askari H, Heidari M, Sadeghian SY, Ober ES, et al. Proteome analysis of sugar beet leaves under drought stress. Proteomics 2005;5:950-60.

19. Hashimoto M, Kisseleva L, Sawa S, Furukawa T, Komatsu S, Koshiba T. A novel rice PR10 protein, RSOsPR10, specifically induced in roots by biotic and abiotic stresses, possibly via the jasmonic acid signaling pathway. Plant Cell Physiol 2004;45:550 9.

20. Hashimoto M, Komatsu S. Proteomic analysis of rice seedlings during cold stress. Proteomics 2007;7:1293-302.

21. Huang J, Wang MM, Jiang Y, Bao YM, Huang X, Sun H, et al. Expression analysis of rice A20/AN1-type zinc finger genes and characterization of ZFP177 that contributes to temperature stress tolerance. Gene 2008;420:135-44.

22. Imin N, Kerim T, Rolfe BG, Weinman JJ. Effect of early cold stress on the maturation of rice anthers. Proteomics 2004;4:1873-82.

23. Imin N, Kerim T, Weinman JJ, Rolfe BG. Low temperature treatment at the young microspore stage induces protein changes in rice anthers. Mol Cell Proteomics 2006;5:274-92.

24. Khan M, Takasaki H, Komatsu S. Comprehensive phosphoproteome analysis in rice and identification of phosphoproteins responsive to different hormones/stresses. J Proteome Res 2005;4:1592-9.

25. Kikuchi S, Satoh K, Nagata T, Kawagashira N, et al. Collection, mapping, and annotation of over 28000 cDNA clones from japonica rice. Science 2003;301:376-9.

26. Kim DW, Rakwal R, Agrawal GK, Jung YH, Shibato J, Jwa NS, et al. A hydroponic rice seedling culture model system for investigating proteome of salt stress in rice leaf. Electrophoresis $2005 ; 26: 4521-39$.

27. Kim KM, Kwon YS, Lee JJ, Eun MY, Sohn JK. QTL mapping and molecular marker analysis for the resistance of rice to ozone. Mol Cells 2004;17:151-5.

28. Komatsu S. Rice proteome database: a step toward functional analysis of the rice genome. Plant Mol Biol 2005;59:179-90.

29. Komatsu S. Plasma membrane proteome in Arabidopsis and rice. Proteomics (on line);2008.

30. Komatsu S, Karibe H, Hamada T, Rakwal R. Phosphorylation upon cold stress in rice (Oryza sativa L.) seedlings. Theor Appl Genet 1999;98:1304-10.

31. Komatsu S, Kojima K, Suzuki K, Ozaki K, Higo K. Rice proteome database based on two-dimensional polyacrylamide gel electrophoresis: its status in 2003. Nucl Acids Res 2004;32:388-92.

32. Komatsu S, Konishi H, Shen S, Yang G. Rice proteomics: a step toward functional analysis of the rice genome. Mol Cell Proteomics $2003 ; 2: 2-10$.

33. Komatsu S, Yamada E, Furukawa K. Cold stress changes the concanavalin A-positive glycosylation pattern of proteins expressed in the basal parts of rice leaf sheaths. Amino Acids (on line);2008.

34. Komatsu S, Yang G, Khan M, Onodera H, Toki S, Yamaguchi M. Over-expression of calcium-dependent protein kinase 13 and calreticulin interacting protein 1 confers cold tolerance on rice plants. Mol Genet Genomics 2007;277:713-23.

35. Komatsu S, Yano H. Update and challenges on proteomics in rice. Proteomics 2006;6:4057-68.

36. Kwon SJ, Choi EY, Choi YJ, Ahn JH, Park OK. Proteomics studies of post-translational modifications in plants. J Exp Bot 2006;57:1547-51.

37. Lee DG, Ahsan N, Lee SH, Kang KY, Lee JJ, Lee BH. An approach to identify cold-induced low-abundant proteins in rice leaf. C R Biol 2007;330:215-25.

38. Lee DG, Ahsan N, Lee SH, Lee JJ, Bahk JD, Kang KY, et al. Chilling stress-induced proteomic changes in rice roots. J Plant Physiol. 2008;(on line).
39. Lyons JM. Chilling injury in plants. Annu Rev Plant Physiol 1973;24:445-66.

40. Morgante M, Salamini F. From plant genomics to breeding practice. Curr Opin Biotechnol 2003;14:214-9.

41. Nakashima K, Tran LS, Van Nguyen D, Fujita M, Maruyama K, Todaka D, et al. Functional analysis of a NAC-type transcription factor OsNAC6 involved in abiotic and biotic stress-responsive gene expression in rice. Plant J 2007;51:617-30.

42. Nohzadeh Malakshah S, Habibi Rezaei M, Heidari M, Salekdeh GH. Proteomics reveals new salt responsive proteins associated with rice plasma membrane. Biosci Biotechnol Biochem 2007;71:2144-54.

43. Nuhse TS, Bottrill AR, Jones AME, Peck SC. Quantitative phosphoproteomic analysis of plasma membrane proteins reveals regulatory mechanism of plant innate responses. Plant J 2007;51:931-40.

44. Parker R, Flowers TJ, Moore AL, Harpham NV. An accurate and reproducible method for proteome profiling of the effects of salt stress in the rice leaf lamina. J Exp Bot 2006;575:1109-18.

45. Qureshi MI, Qadir S, Zolla L. Proteomics-based dissection of stress-responsive pathways in plants. J Plant Physiol 2007;164:1239-60.

46. Rabbani MA, Maruyama K, Abe H, Khan MA, Katsura K, Ito Y, et al. Monitoring expression profiles of rice genes under cold, drought, and high-salinity stresses and abscisic acid application using cDNA microarray and RNA gel-blot analyses. Plant Physiol 2003;133:1755-67.

47. Raison JK, Lyons JM, Keith AD. Temperature-induced phase changes in mitochondrial membranes detected by spin labeling. J Biol Chem 1971;246:4036-40.

48. Sahi C, Singh A, Kumar K, Blumwald E, Grover A. Salt stress response in rice: genetics, molecular biology, and comparative genomics. Funct Integr Genomics 2006;6:263-84.

49. Salekdeh GH, Siopongco J, Ghareyazie B, Bennett J. Proteomic analysis of rice leaves during drought stress and recovery. Proteomics 2002;2:1131-45.

50. Salekdeh GH, Komatsu S. Crop proteomics: aim at sustainable agriculture of tomorrow. Proteomics 2007;7:2976-96.

51. Sanchez PA, Swaminathan MS. Public health. Cutting world hunger in half. Science 2005;307:357-9.

52. Shen S, Jing Y, Kuang T. Proteomics approach to identify woundresponse related proteins from rice leaf sheath. Proteomics 2003;3:527-35.

53. The International Rice Genome Sequencing Project. The mapbased sequence of the rice genome. Nature 2005;436:793-800.

54. The Rice Annotation Project. Curated genome annotation of Oryza sativa ssp. japonica and comparative genome analyusis with Arabidopsis thaliana. Genome Res 2007;17:175-83.

55. Wienkoop S, Glinski M, Tanaka N, Tolstikov V, Fiehn O, Weckwerth W. Linking protein fractionation with multidimensional monolithic reversed-phase peptide chromatography/mass spectrometry enhances protein identification from complex mixtures even in the presence of abundant proteins. Rapid Commun Mass Spectrom 2004;18:643-50.

56. Wulfkuhle JD, Paweletz CP, Steeg PS, Petricoin EF, Liotta L. Proteomic approaches to the diagnosis, treatment, and monitoring of cancer. Adv Exp Med Biol 2003;532:59-68.

57. Yan S, Tang Z, Su W, Sun W. Proteomic analysis of salt stressresponsive proteins in rice root. Proteomics 2005;5:235-44.

58. Yan SP, Zhang QY, Tang ZC, Su WA, Sun WN. Comparative proteomic analysis provides new insights into chilling stress responses in rice. Mol Cell Proteomics 2006;53:484-96.

59. Zang X, Komatsu S. A proteomics approach for identifying osmotic-stress-related proteins in rice. Phytochem 2007;68:42637. 